\title{
CONGENITAL THROMBOCYTOPENIC PURPURA TREATED BY EXCHANGE TRANSFUSION
}

\author{
BY \\ J. M. BRIDGES and I. J. CARRÉ \\ From the Department of Clinical Pathology, Royal Victoria Hospital, Belfast and \\ the Department of Child Health, The Queen's University of Belfast
}

(RECEIVED FOR PUBLICATION JUNE 7, 1960)

The occurrence of thrombocytopenic purpura in babies born to mothers with idiopathic thrombocytopenia is a well recognized though uncommon clinical entity. Robson and Walker (1951) were able to collect from the literature only 19 definite and nine probable examples of this association.

The suggestion of Epstein, Lozner, Cobbey and Davidson (1950) that this type of congenital thrombocytopenic purpura results from the transfer across the placenta of a maternal factor capable of causing thrombocytopenia has recently received support with the demonstration of platelet agglutinins in the serum of both mother and child (Harrington, Sprague, Minnich, Moore, Aulvin and Dubach, 1953; Vanderbroucke and Verstraete, 1955; Tullis, 1956; Schoen, King and Duane, 1956). In view of these findings it might be expected that exchange transfusion of affected infants would, by removing the acquired platelet antibodies, shorten the period of thrombocytopenia and so lessen the risk of haemorrhage and death. We report below observations on a baby with congenital thrombocytopenic purpura who was treated in this manner.

\section{Case Report}

C.B., now aged 30 , had a splenectomy performed when 10 years old for a persistent haemorrhagic disorder regarded as idiopathic thrombocytopenic purpura. Since that time she has had no episodes of excessive bleeding and additionally has had many teeth extracted without trouble.

In June 1958, after an uneventful antenatal period and confinement she was delivered of a female child weighing $6 \mathrm{lb} .3 \mathrm{oz}$. Shortly after birth the baby was noticed to have purpura which became more widespread during the 14 hours of its survival. Necropsy revealed no haemorrhages other than those in skin; an extensive pneumonia was present and thought to have been the cause of death.

The mother's blood was examined postnatally (August 1958) with the following results: $\mathrm{Hb} 13.3 \mathrm{~g} . / 100 \mathrm{ml}$., packed cell volume $43 \%$, white cell count $7,100 /$ c.mm., platelets $124,000 /$ c.mm., bleeding time $7 \mathrm{~min}$, clotting time $4 \mathrm{~min}$. $45 \mathrm{sec}$. (ordinary glass), $6 \mathrm{~min}$. (silicone glass), clot retraction normal in one hour, plasma prothrombin $11 \mathrm{sec} .=100 \%$ (control $11 \mathrm{sec} .=100 \%)$, fibrinogen $137 \mathrm{mg}$. \% and thromboplastin generation normal. The platelet count was done by the direct method using Rees Ecker diluting fluid on a venous sample of blood, as were all platelet counts on the mother; the normal values for the platelet count as done by this method, are taken as $200-400,000 / \mathrm{c} . \mathrm{mm}$.

Her blood was next examined in July 1959, at which time she was six months pregnant. On that occasion the platelets numbered 196,000/c.mm.; all the other previous blood tests were repeated and found to be within normal limits. On October 7 , when 39 weeks pregnant, her platelet count was only $84,000 /$ c.mm.; as before, all the other blood tests showed no abnormality. Although thrombocytopenic there was no clinical evidence of any bleeding tendency. On October 22, after an uneventful pregnancy and confinement, she was delivered of a male child weighing $7 \mathrm{lb} .11 \mathrm{oz}$. The puerperium was uneventful. On the third postnatal day the mother's platelet count was $120,000 /$ c.mm.; three months later it was $128,000 /$ c.mm.

The baby, when examined shortly after birth, was found to have a number of purpuric spots on the face and trunk; no other abnormalities were noted. Examination of blood obtained from the baby when 2 hours old gave the following results: $\mathrm{Hb} 17 \cdot 2 \mathrm{~g} . / 100 \mathrm{ml}$., platelets $34,000 /$ c.mm., leucocytes $18,200 /$ c.mm. with a differential white cell count of neutrophils $71 \%$, lymphocytes $27 \%$ and monocytes $2 \%$; the bleeding time was $8 \mathrm{~min}$. $30 \mathrm{sec}$. Examination of a stained film confirmed the lack of platelets: those present showed no morphological abnormality. Blood samples for these and other studies on the baby were obtained by heel prick unless otherwise stated.

When 3 hours old the baby was given an exchange transfusion with $520 \mathrm{ml}$. ' $O$ ' Rhesus negative blood which had been collected during the preceding 24 hours. During the transfusion, which was well tolerated, fresh purpuric spots and ecchymoses appeared mostly on the face. Further fresh crops of purpura were noted during the next three days but not thereafter. Following transfusion he was started on oral cortisone: $25 \mathrm{mg}$. were given during the next 24 hours followed by $8 \mathrm{mg}$. 


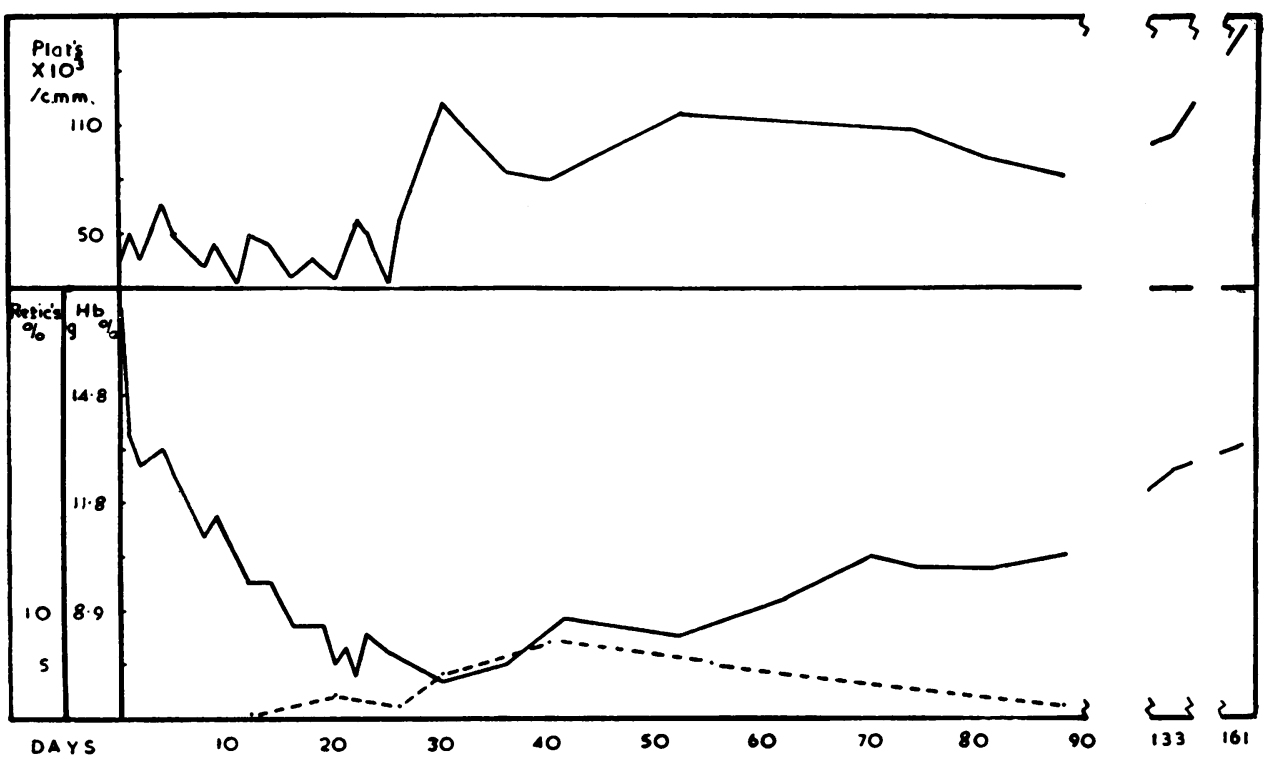

FIG. 1.-Platelet count, haemoglobin level and reticulocyte count during first five months.

daily for four days and $4 \mathrm{mg}$. daily for five weeks; the dose was then gradually reduced, therapy being discontinued after a further six weeks.

On the day after exchange transfusion the bleeding time had fallen to 6 min. 30 sec., 10 days later it was $5 \mathrm{~min}$. and on all subsequent occasions was less than $4 \mathrm{~min}$. There was, however, no commensurate increase in the platelet count which during the first month never exceeded $80,000 /$ c.mm. Between the first and third months the platelet count varied between 80,000 and $120,000 /$ c.mm. At 4 months the baby's platelet count was still only $106,000 /$ c.mm., but at 5 months this had risen to 166,000 (Fig. 1).

Specimens of venous blood obtained from the baby immediately before and after exchange transfusion and from the mother on the day following delivery were examined for platelet agglutinins by the method described by Kissmeyer-Nielsen (1955). The mother's serum and the baby's pre-transfusion serum both gave a strong positive result whereas the post-transfusion serum from the baby gave only a doubtful positive result. Twentysix days after birth when the baby's platelet count was still only $59,000 / \mathrm{c} . \mathrm{mm}$. both the mother's and baby's sera were again examined for platelet agglutinins. On this occasion the mother's serum gave a strong positive result as previously whereas the baby's was definitely negative.

From a post-transfusion level of $13.7 \mathrm{~g} . / 100 \mathrm{ml}$. the baby's $\mathrm{Hb}$ fell steadily to $7 \cdot 2 \mathrm{~g}$. $/ 100 \mathrm{ml}$. with $1 \%$ reticulocytes by the 26th day. Two weeks later the $\mathrm{Hb}$ was still only $7 \cdot 4 \mathrm{~g} . / 100 \mathrm{ml}$. but the reticulocytes had increased to $7 \%$. Thereafter there was a gradual rise in $\mathrm{Hb}$ to $13.3 \mathrm{~g} . / 100 \mathrm{ml}$. at 5 months (Fig. 1).

A sample of bone marrow was aspirated from the iliac crest on the 27th day. Though the sample was diluted with sinusoidal blood the relative number of normoblasts appeared somewhat reduced; otherwise no abnormality was noted and, in particular, appreciable numbers of morphologically normal megakaryocytes were seen.

Except for the development of purpura during the first three days the baby showed no other signs of bleeding. His clinical progress was wholly uneventful, he was bottle-fed throughout and at 3 months weighed $13 \mathrm{lb} .12 \mathrm{oz}$.

\section{Discussion}

It is now established that neonatal thrombocytopenic purpura is a clinical disorder of multiple aetiology (Kaplan, 1959). One form is that which occurs in babies born to mothers suffering from idiopathic thrombocytopenic purpura. Platelet agglutinins have now been demonstrated in both the serum of the mother and of her thrombocytopenic infant on at least five occasions (Harrington et al., 1953; Vanderbroucke and Verstraete, 1955; Tullis, 1956; Schoen et al., 1956, and the present case) thereby providing good reason for believing the disorder to be due to the passive transfer of maternal antibodies.

This type of congenital thrombocytopenic purpura is a self-limiting condition. Complete recovery can be expected provided affected infants survive the hazards of haemorrhage during the early neonatal period. Robson and Walker (1951) and Morris (1954) found that of those infants reported in the literature $15-20 \%$ had died within the first 
week. The severity of the disease in the mother and the sex of the child are two factors which appear to affect the chances of survival. In general, the more active the disease in the mother the more likely is the baby to be badly affected. There have, however, been reports of affected infants being born to mothers who were either in spontaneous remission or, as in the present case, were clinically well following splenectomy (Robson and Davidson, 1950; Epstein et al., 1950). Secondly, Robson and Walker (1951) in their review of published cases, noted that although slightly more female children were affected all fatalities had occurred in males.

Although fresh purpuric lesions usually cease to appear between the third and 10th day the bleeding time in most cases remains prolonged for some 10 to 28 days. Thrombocytopenia usually persists even longer commonly up to six but rarely beyond 15 weeks with an average duration of the order of 10 weeks (Harrington et al., 1953; Morris, 1954; Vanderbroucke and Verstraete, 1955; Stefanini and Dameshek, 1955).

Only the reports of Schoen et al. (1956) and Vanderbroucke and Verstraete (1955) mention the length of time the platelet agglutinins persist in the foetal circulation and only in the latter is this correlated with the platelet count and bleeding time. Schoen et al. found platelet agglutinins to be present at 38 but absent at 80 days. Vanderbroucke and Verstraete record a positive result for platelet agglutinins at 39 days with a negative result at 78 days. The platelet count in their patient at these times was 100,000 and $110,000 / \mathrm{c} . \mathrm{mm}$.; four weeks later when the baby was 15 weeks old the platelets numbered $260,000 / \mathrm{c} . \mathrm{mm}$. The bleeding time which on the first day was $22 \mathrm{~min}$. had fallen to $1 \frac{1}{2} \mathrm{~min}$. by the 11 th day. On the basis of these findings it seems that thrombocytopenia is likely to persist for some time after the disappearance of the platelet agglutinins. This is contrary to the view of Kaplan (1959) who without reference to specific examples records that platelet antibodies may persist after restoration of the platelet count to normal.

In view of the risk to life from haemorrhage during the first week, treatment of these infants must be directed primarily at preventing such catastrophes. No treatment has yet proved effective (Killander, 1959). If one accepts that the thrombocytopenia in the infant is due to the passive transfer of maternal platelet agglutinins then it seems logical to treat these infants by exchange transfusion with the object of washing out the transmitted maternal antibodies. In our patient, no new purpuric lesions appeared after three days, the bleeding time fell to less than $4 \mathrm{~min}$. from the 10th day onwards and the platelet count, initially $34,000 /$ c.mm., remained below $80,000 /$ c.mm. until the 29 th day and was still only $166,000 / \mathrm{c} . \mathrm{mm}$. at 5 months. Platelet agglutinins present before the start of the exchange transfusion could no longer be identified with certainty immediately after the transfusion and were definitely absent on re-examination of the baby's serum at 26 days. The wide variation in the natural history and the paucity of data relating to the persistence of platelet antibodies make it impossible to assess accurately the value of such therapy by reference to only a single case. Comparison with the reports of Vanderbroucke and Verstraete and Schoen et al. suggests that we did succeed in accelerating the elimination of antibodies from the baby's serum. However, there is nothing to indicate that this had any effect in shortening the duration of purpura, prolonged bleeding time and thrombocytopenia. These observations are similar to those of Killander (1959) who alone has treated a similar case by exchange transfusion. In this author's patient bleeding episodes occurred up to 4 weeks and the platelets still numbered only $60,000 / \mathrm{c} . \mathrm{mm}$. at 7 weeks.

It is of interest that in both our patient and that of Killander exchange transfusion was followed by a period of anaemia. In the present case there was no evidence to suggest a haemolytic process as the cause of the anaemia; generalized marrow failure can also be excluded as an aetiological factor on the basis of the essentially normal marrow picture. Thus it appears likely that the anaemia was of similar origin to that which not uncommonly occurs after exchange transfusion for haemolytic disease especially when the immediate post-transfusion haemoglobin level is less than $15 \mathrm{~g} . / 100 \mathrm{ml}$. (Gairdner, 1958) as was the case with our patient.

This apparent lack of response to exchange transfusion in congenital thrombocytopenia is in marked contrast to that recorded by Stefanini, Mednicoff and Plitman (1954) on a baby with erythroblastosis foetalis and associated thrombocytopenia. Maternal platelet iso-agglutinins were detected in the baby's serum. After the second of two exchange transfusions performed within 48 hours of birth the purpura disappeared and the platelet count rose promptly from 60,000 to 250,000 / c.mm. subsequently remaining above this figure. This type of response is what one might expect if the baby's low platelet count was due solely to the destruction of platelets by circulating antibody. Is the lack of a similar response in babies born to mothers with idiopathic thrombocytopenic purpura indicative of a more complex mechanism? Some support for this view is provided by the observation 
that the thrombocytopenia may persist for a considerable time after the disappearance of antibodies (Vanderbroucke and Verstraete, 1955; and the present case).

Corticosteroids are of acknowledged value in the treatment of patients suffering from idiopathic thrombocytopenic purpura and it therefore seems logical to use these drugs on babies with congenital thrombocytopenic purpura. As judged by the rate of improvement in the bleeding time and platelet count cortisone did not appear to hasten the recovery of our patient nor that of Schoen et al. (1956). This is not to say that corticosteroids may not be beneficial in this condition for in idiopathic thrombocytopenic purpura it is recognized that cortisone lessens the risk of haemorrhage often without producing any effect on the platelet count. In view of the not inconsiderable risks of haemorrhage during the first week of life it seems desirable to treat these infant patients with corticosteroids.

The result of exchange transfusion in our patient is disappointing and suggests that this procedure may not materially alter the natural course of the disease. A final assessment of the therapeutic value of both exchange transfusion and corticosteroids must, however, await the publication of detailed studies on more patients.

\section{Summary}

A case report of a baby with congenital thrombocytopenic purpura is presented. The baby's mother had had a splenectomy for idiopathic thrombocytopenic purpura 20 years previously. The infant was treated by exchange transfusion and corticosteroids.

Platelet agglutinins which were demonstrated in the serum of mother and baby shortly after birth could not be identified in the baby's serum at 26 days. The thrombocytopenia, however, persisted and during the first three months the platelet count never rose above $120,000 /$ c.mm.

Although the baby made an uneventful clinical recovery it is doubtful if therapy materially altered the natural course of the condition.

We should like to thank Professor F. M. B. Allen and Dr. M. G. Nelson for their interest and advice in preparing this report; Professor C. H. G. Macafee, Mr. M. R. Neely and Dr. J. H. Morison for access to case records of their patients; and Mr. D. Boyd for technical assistance.

\section{REFERENCES}

Epstein, R. D., Lozner, E. L., Cobbey, T. S., Jr. and Davidson, C. S. (1950). Congenital thrombocytopenic purpura. Purpura hemorrhagica in pregnancy and in the newborn. Amer. J. Med., 9,44 .

Gairdner, D. (1958). Recent Advances in Paediatrics, p. 72. Churchill, London.

Harrington, W. J., Sprague, C. C., Minnich, V., Moore, C. V., Aulvin, R. C. and Dubach, R. (1953). Immunologic mechanisms in idiopathic and neonatal thrombocytopenic purpura. Ann. intern. Med., 38, 433.

Kaplan, E. (1959). Congenital and neonatal thrombocytopenic purpura. J. Pediat., 54, 644.

Killander, A. (1959). On the use of exchange transfusion in neonatal thrombocytopenic purpura. Acta paediat. (Uppsala), 48,
Suppl. 117, 29.

Kissmeyer-Nielsen, F. (1955). Demonstration of platelet antibodies by haemagglutination of antigen-coated tanned erythrocytes. Sang, 26, 117.

Sang, 26, 117.
Morris, M. B. (1954). Thrombocytopenic purpura in the newborn. Arch. Dis. Childh., 29, 75.

Robson, H. N. and Davidson, L. S. P. (1950). Purpura in pregnancy with special reference to idiopathic thrombocytopenic purpura. Lancet, 2, 164.

and Walker, C. H. M. (1951). Congenital and neonatal thrombocytopenic purpura. Arch. Dis. Childh., 26, 175.

Schoen, E. J., King, A. L. and Duane, R. T. (1956). Neonatal thrombocytopenic purpura. Pediatrics, 17, 72.

Stefanini, M. and Dameshek, W. (1955). The Hemorrhagic Disorders p. 107. Grune and Stratton, New York.

, Mednicoff, I. B. and Plitman, G. I. (1954). Neonatal thrombocytopenia. Int. Rec. Med., 167, 27.

Tullis, J. L. (1956). Identification and significance of platelet antibodies. New Engl. J. Med., 255, 541.

Vanderbroucke, J. and Verstraete, M. (1955). Thrombocytopenia due to platelet agglutinins in the newborn. Lancet, 1, 593. 